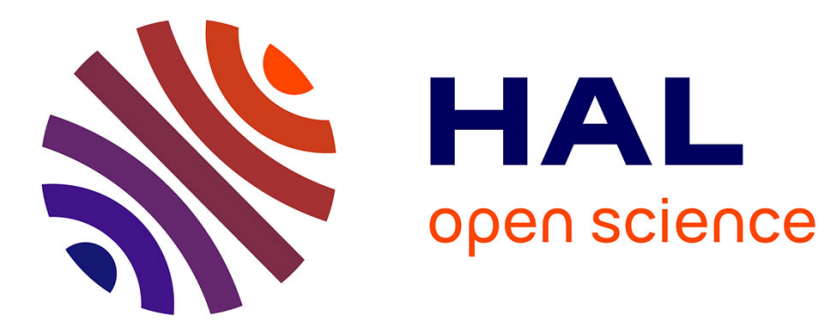

\title{
Generalized plasmas and the anomalous quantum Hall effect
}

\author{
P.J. Forrester, B. Jancovici
}

\section{To cite this version:}

P.J. Forrester, B. Jancovici. Generalized plasmas and the anomalous quantum Hall effect. Journal de Physique Lettres, 1984, 45 (12), pp.583-589. 10.1051/jphyslet:019840045012058300 . jpa-00232385

\section{HAL Id: jpa-00232385 https://hal.science/jpa-00232385}

Submitted on 1 Jan 1984

HAL is a multi-disciplinary open access archive for the deposit and dissemination of scientific research documents, whether they are published or not. The documents may come from teaching and research institutions in France or abroad, or from public or private research centers.
L'archive ouverte pluridisciplinaire HAL, est destinée au dépôt et à la diffusion de documents scientifiques de niveau recherche, publiés ou non, émanant des établissements d'enseignement et de recherche français ou étrangers, des laboratoires publics ou privés. 
Classification

Physics Abstracts

$05.20-72.20 \mathrm{M}$

\title{
Generalized plasmas and the anomalous quantum Hall effect
}

\author{
P. J. Forrester \\ Department of Theoretical Physics, Research School of Physical Sciences, \\ The Australian National University, Canberra, 2601 Australia \\ and B. Jancovici \\ Laboratoire de Physique Théorique et Hautes Energies $\left({ }^{*}\right)$, Université Paris-Sud, \\ 91405 Orsay Cedex, France
}

(Reçu le 2 avril 1984, accepté le 24 avril 1984)

\begin{abstract}
Résumé. - A cause d'une analogie mathématique, il pourrait y avoir une relation entre l'effet Hall quantique anormal et la mécanique statistique classique d'un plasma bidimensionnel généralisé : il s'agit d'un système bidimensionnel de deux espèces de particules, interagissant par des potentiels logarithmiques, avec trois constantes de couplage indépendantes (au lieu de deux charges pour un plasma ordinaire à deux composantes). On établit des règles de somme pour de tels systèmes. On étudie un modèle soluble unidimensionnel et on montre qu'il obéit aux analogues unidimensionnels de ces règles de somme.
\end{abstract}

\begin{abstract}
Because of a mathematical analogy, the anomalous quantum Hall effect might be related to the classical statistical mechanics of a two-dimensional generalized plasma : this is a two-dimensional system of two species of particles, interacting through logarithmic potentials, with three independent coupling constants (instead of two charges for an ordinary two-component plasma). Sum rules are derived for such systems. A one-dimensional solvable model is studied and shown to obey the one-dimensional analogs of the sum rules.
\end{abstract}

\section{Introduction.}

The experimental discovery [1, 2] of the anomalous quantum Hall effect (quantization of the Hall resistance of a two-dimensional electron gas in a high magnetic field, for a Landau level fractional filling) has induced an intense theoretical activity, following an important paper by Laughlin [3]. A variety of trial wave functions has been considered for these systems. One of these wave functions, proposed by Halperin [4], assumes that there are both spin up electrons (roman indices) and spin down electrons (greek indices) well described by a wave function of the form

$$
\begin{aligned}
& \Psi=\prod_{i>j}\left(z_{i}-z_{j}\right)^{\frac{1}{2} g_{\mathrm{RR}}} \prod_{\alpha>\beta}\left(z_{\alpha}-z_{\beta}\right)^{\frac{1}{2^{g} g_{\mathrm{GG}}}} \prod_{i, \alpha}\left(z_{i}-z_{\alpha}\right)^{\frac{1}{2} g_{\mathrm{RG}}} \times \\
& \times \exp \left\{-\left[\sum_{i}\left|z_{i}\right|^{2}+\sum_{\alpha}\left|z_{\alpha}\right|^{2}\right] / 4 l^{2}\right\},
\end{aligned}
$$

(*) Laboratoire associé au Centre National de la Recherche Scientifique. 
where the complex number $z_{i}=x_{i}-i y_{i}$ describes the cartesian coordinates $\left(x_{i}, y_{i}\right)$ of the $i$ th electron; $l$ is the Landau magnetic length. According to Halperin [4], a possible set of exponents would be for instance $\frac{1}{2} g_{\mathrm{RR}}=\frac{1}{2} g_{\mathrm{GG}}=3, \frac{1}{2} g_{\mathrm{RG}}=2$; anyhow these exponents have to be positive.

A remarkable feature of (1.1) is that the corresponding many-body density $|\Psi|^{2}$ has the same form as the Boltzmann factor for a classical "generalized" two-dimensional binary plasma, made of two species (roman and greek) of particles interacting through logarithmic potentials. For two particles at a distance $r$ from one another, the interaction potential is $-g_{\mathrm{RR}} \ln r$ for a roman pair, $-g_{\mathrm{GG}} \ln r$ for a greek pair, $-g_{\mathrm{RG}} \ln r$ for a roman-greek pair; furthermore, the particles are immersed in a circular uniform background creating a potential $\left|z_{i}\right|^{2} / 2 l^{2}$ upon particle $i$ and $\left|z_{\alpha}\right|^{2} / 2 l^{2}$ upon particle $\alpha$. Then, at the inverse temperature $\beta=1$, the Boltzmann factor of this classical system is precisely $|\Psi|^{2}$. Thus, studying the classical system, and especially its pair distribution functions, might be of interest for the anomalous quantum Hall effect.

Following Girvin [5], we call the classical system a " generalized " plasma, because the usual Coulomb relation between the coupling constants, i.e. $g_{\mathrm{RR}} g_{\mathrm{GG}}-g_{\mathrm{RG}}^{2}=0$, is not necessarily satisfied.

In this Letter, we extend sum rules given by Girvin [5]; we show that the distribution functions of a generalized two-dimensional plasma obeys not only screening rules, but also generalizations of the Stillinger-Lovett [6] rule, which determine the second moments of the correlation functions. Furthermore, as a check and illustration, we describe a one-dimensional solvable model which does obey the one-dimensional analogs of the sum rules.

\section{Two-dimensional generalized plasma.}

For a system of roman and greek particles of number densities $\rho_{\mathrm{R}}$ and $\rho_{\mathrm{G}}$ respectively, the Hamiltonian is

$$
\begin{aligned}
H=-g_{\mathrm{RR}} & \sum_{i>j} \ln r_{i j}-g_{\mathrm{GG}} \sum_{\alpha>\beta} \ln r_{\alpha \beta}-g_{\mathrm{RG}} \sum_{i, \alpha} \ln r_{i \alpha} \\
& +\left(g_{\mathrm{RR}} \rho_{\mathrm{R}}+g_{\mathrm{RG}} \rho_{\mathrm{G}}\right) \sum_{i} \int \ln \left|\mathbf{r}_{i}-\mathbf{r}\right| \mathrm{d} \mathbf{r}+\left(g_{\mathrm{GG}} \rho_{\mathrm{G}}+g_{\mathrm{RG}} \rho_{\mathrm{R}}\right) \sum_{\alpha} \int \ln \left|\mathbf{r}_{\alpha}-\mathbf{r}\right| \mathrm{d} \mathbf{r}- \\
& -\left(\frac{1}{2} g_{\mathrm{RR}} \rho_{\mathrm{R}}^{2}+\frac{1}{2} g_{\mathrm{GG}} \rho_{\mathrm{G}}^{2}+g_{\mathrm{RG}} \rho_{\mathrm{R}} \rho_{\mathrm{G}}\right) \int \ln \left|\mathbf{r}-\mathbf{r}^{\prime}\right| \mathrm{d} \mathbf{r} \mathbf{d r}^{\prime},
\end{aligned}
$$

where the particle-background and background-background interactions have been chosen in a way which compensates the remote particle-particle interactions; thus we expect the system to have a well-behaved thermodynamic limit. The particle-background interaction in (2.1) can be rewritten, for a circular background, as a constant plus

$$
\left(g_{\mathrm{RR}} \rho_{\mathrm{R}}+g_{\mathrm{RG}} \rho_{\mathrm{G}}\right) \frac{\pi}{2} \sum_{i} r_{i}^{2}+\left(g_{\mathrm{GG}} \rho_{\mathrm{G}}+g_{\mathrm{RG}} \rho_{\mathrm{R}}\right) \frac{\pi}{2} \sum_{\alpha} r_{\alpha}^{2}
$$

[(1.1) is recovered [5] by choosing $\pi \rho_{\mathrm{R}} l^{2}=\left(g_{\mathrm{GG}}-g_{\mathrm{RG}}\right) / \Delta$ and $\pi \rho_{\mathrm{G}} l^{2}=\left(g_{\mathrm{RR}}-g_{\mathrm{RG}}\right) / \Delta$ with $\Delta=g_{\mathrm{RR}} g_{\mathrm{GG}}-g_{\mathrm{RG}}^{2}$. $]$

The correlation functions $h_{\mathrm{AB}}(r)$ (where A, B stand for $\mathrm{R}$ or $\mathrm{G}$ ) are defined in such a way that, when a particle $\mathrm{A}$ is at the origin, the density of particles $\mathrm{B}$ at $\mathbf{r}$ is $\rho_{\mathrm{B}}\left[1+h_{\mathrm{AB}}(r)\right]$. We define dimensionless Fourier transforms

$$
\hat{h}_{\mathrm{AB}}(k)=\left(\rho_{\mathrm{A}} \rho_{\mathrm{B}}\right)^{1 / 2} \int h_{\mathrm{AB}}(r) \exp (i \mathbf{k} \cdot \mathbf{r}) \mathrm{dr} .
$$


We introduce direct correlation functions $c_{\mathrm{AB}}(r)$, the Fourier transforms of which,

$$
\hat{c}_{\mathrm{AB}}(k)=\left(\rho_{\mathrm{A}} \rho_{\mathrm{B}}\right)^{1 / 2} \int c_{\mathrm{AB}}(r) \exp (i \mathbf{k} \cdot \mathbf{r}) \mathrm{d} \mathbf{r}
$$

are defined from the $h_{\mathrm{AB}}$ by the Ornstein-Zernicke relations

$$
\hat{h}_{\mathrm{AB}}=\hat{c}_{\mathrm{AB}}+\sum_{\mathrm{C}} \hat{h}_{\mathrm{AC}} \hat{c}_{\mathrm{CB}} \text {. }
$$

Solving (2.4) for the $\hat{h}_{\mathrm{AB}}$ (and assuming the symmetry $\hat{c}_{\mathrm{RG}}=\hat{c}_{\mathrm{GR}}$ ), one finds

$$
1+\hat{h}_{\mathrm{RR}}=\frac{1-\hat{c}_{\mathrm{GG}}}{D}, 1+\hat{h}_{\mathrm{GG}}=\frac{1-\hat{c}_{\mathrm{RR}}}{D}, \hat{h}_{\mathrm{RG}}=\hat{h}_{\mathrm{GR}}=\frac{\hat{c}_{\mathrm{RG}}}{D},
$$

where

$$
D=\left(1-\hat{c}_{\mathrm{RR}}\right)\left(1-\hat{c}_{\mathrm{GG}}\right)-\hat{c}_{\mathrm{RG}}^{2} .
$$

Sum rules for the $h_{\mathrm{AB}}$ are now obtained from the usual assumption [7] that $c_{\mathrm{AB}}(r)$ behaves for large $r$ like $-\beta$ times the potential, or equivalently that, for small $k$,

$$
\hat{c}_{\mathrm{AB}}(k)=-\frac{2 \pi \beta\left(\rho_{\mathrm{A}} \rho_{\mathrm{B}}\right)^{1 / 2} g_{\mathrm{AB}}}{k^{2}}+\hat{c}_{\mathrm{AB}}^{0}(k),
$$

where $\hat{c}_{\mathrm{AB}}^{0}(k)$ is a regular function of $k^{2}$. Using (2.7) in (2.6) and (2.5), for a generalized plasma $\left(\Delta=g_{\mathrm{RR}} g_{\mathrm{GG}}-g_{\mathrm{RG}}^{2} \neq 0\right)$, one finds the small- $k$ behaviours

$$
1+\hat{h}_{\mathrm{RR}}(k) \sim \frac{g_{\mathrm{GG}}}{2 \pi \beta \rho_{\mathrm{R}} \Delta} k^{2}, 1+\hat{h}_{\mathrm{GG}}(k) \sim \frac{g_{\mathrm{RR}}}{2 \pi \beta \rho_{\mathrm{G}} \Delta} k^{2}, \hat{h}_{\mathrm{RG}}(k) \sim \frac{-g_{\mathrm{RG}}}{2 \pi \beta\left(\rho_{\mathrm{R}} \rho_{\mathrm{G}}\right)^{1 / 2} \Delta} k^{2} .
$$

Since $1+\hat{h}_{\mathrm{AA}}(k)$ is the average value of a squared density amplitude, it must be positive; therefore (2.8) is acceptable only if $\Delta>0$, a condition we shall assume to hold (presumably, for $\Delta<0$, the roman-greek repulsion is too strong and the system becomes unstable, perhaps undergoing a phase separation). From (2.8) we recover Girvin's screening rule [5]

$$
\rho_{\mathrm{B}} \int h_{\mathrm{AB}}(r) \mathrm{d} \mathbf{r}=-\delta_{\mathrm{AB}}
$$

and we also find second-moment rules

$$
\rho_{\mathrm{R}}^{2} \int h_{\mathrm{RR}}(r) r^{2} \mathrm{dr}=-\frac{4 g_{\mathrm{GG}}}{2 \pi \beta \Delta}, \rho_{\mathrm{G}}^{2} \int h_{\mathrm{GG}}(r) r^{2} \mathrm{dr}=-\frac{4 g_{\mathrm{RR}}}{2 \pi \beta \Delta}, \rho_{\mathrm{R}} \rho_{\mathrm{G}} \int h_{\mathrm{RG}}(r) r^{2} \mathrm{dr}=\frac{4 g_{\mathrm{RG}}}{2 \pi \beta \Delta} .
$$

From (2.9), it is seen that, in a generalized plasma, a particle A is perfectly screened, but only particles of the same species A participate in that screening; from (2.10), it is seen that simple second-moment rules hold separately for each partial correlation function $h_{\mathrm{AB}}$. These results are to be contrasted with what happens for ordinary Coulomb systems $\left(g_{\mathrm{RR}} g_{\mathrm{GG}}-g_{\mathrm{RG}}^{2}=0\right)$ for which the same approach does not give universal sum rules for each $h_{\mathrm{AB}}$ (the analogs of (2.9) and (2.10) would involve non-universal contributions $\left.\hat{c}_{\mathrm{AB}}^{0}(0)\right)$. For ordinary Coulomb systems, one only finds the screening rules involving both species

$$
\sum_{\mathrm{B}} \rho_{\mathrm{B}}\left(\frac{g_{\mathrm{BB}}}{g_{\mathrm{AA}}}\right)^{1 / 2} \int h_{\mathrm{AB}}(r) \mathrm{d} \mathbf{r}=-1
$$


and the total charge-charge Stillinger-Lovett rule [6]

$$
\sum_{\mathrm{A}, \mathrm{B}}\left(g_{\mathrm{AA}} g_{\mathrm{BB}}\right)^{1 / 2} \rho_{\mathrm{A}} \rho_{\mathrm{B}} \int h_{\mathrm{AB}}(r) r^{2} \mathrm{~d} \mathbf{r}=-\frac{4}{2 \pi \beta} .
$$

\section{One-dimensional generalized plasma.}

The considerations of section 2 can be adapted for a one-dimensional system. The interactions are still supposed to be logarithmic and described by (2.1), but now the particles are on a line (it may be convenient to put the particles on a circle rather than on a straight line; this should make no difference in the thermodynamic limit). The analysis is the same as in section 2, except that the Fourier transforms are now one-dimensional, and therefore instead of (2.7) one has

$$
\hat{c}_{\mathrm{AB}}(k)=-\frac{\pi \beta\left(\rho_{\mathrm{A}} \rho_{\mathrm{B}}\right)^{1 / 2} g_{\mathrm{AB}}}{|k|}+c_{\mathrm{AB}}^{0}(k),
$$

and instead of $(2.8)$ one finds

$$
1+\hat{h}_{\mathrm{RR}}(k) \sim \frac{g_{\mathrm{GG}}}{\pi \beta \rho_{\mathrm{R}} \Delta}|k|, \quad 1+\hat{h}_{\mathrm{GG}}(k) \sim \frac{g_{\mathrm{RR}}}{\pi \beta \rho_{\mathrm{G}} \Delta}|k|, \quad \hat{h}_{\mathrm{RG}}(k) \sim-\frac{g_{\mathrm{RG}}}{\pi \beta\left(\rho_{\mathrm{R}} \rho_{\mathrm{G}}\right)^{1 / 2} \Delta}|k|,
$$

where again $\Delta=g_{\mathrm{RR}} g_{\mathrm{GG}}-g_{\mathrm{RG}}^{2}$. The screening rules (2.9) are still valid, but now the Fourier transforms $\hat{h}_{\mathrm{AB}}(k)$ have a cusp at $k=0$, and instead of second-moment rules, one finds that the $h_{\mathrm{AB}}(r)$ have asymptotic expansions, as $r \rightarrow \infty$,

$\rho_{\mathrm{R}}^{2} h_{\mathrm{RR}}(r) \sim-\frac{g_{\mathrm{GG}}}{\pi^{2} \beta \Delta} \frac{1}{r^{2}}+\cdots, \rho_{\mathrm{G}}^{2} h_{\mathrm{GG}}(r) \sim-\frac{g_{\mathrm{RR}}}{\pi^{2} \beta \Delta} \frac{1}{r^{2}}+\cdots, \quad \rho_{\mathrm{R}} \rho_{\mathrm{G}} h_{\mathrm{RG}}(r) \sim \frac{g_{\mathrm{RG}}}{\pi^{2} \beta \Delta} \frac{1}{r^{2}}+\cdots$.

Other singularities on the real axis for finite values of $k$ might occur in the $\hat{h}_{\mathrm{AB}}(k)$, and therefore there might be additional oscillating terms in (3.3).

\section{A solvable one-dimensional model.}

In one dimension, for the special case $\beta g_{\mathrm{RR}}=2, \beta g_{\mathrm{GG}}=4, \beta g_{\mathrm{RG}}=2$, one obtains a solvable model, which had been dormant for some time in the files of one of us (P. J. F.) and which provides an illustration of the rules (2.9) and (3.3). On a circle of radius $R$, we put $a N$ roman and $b N$ greek particles, located by their polar angles $\theta$.

4.1 Partition function AND fReE ENERgy. - The excess partition function obtained from $(2.1)$ is

$$
\begin{aligned}
& Z=(2 \pi)^{-(a+b) N} R^{-(a+2 b) N} \prod_{k=1}^{a N} \int_{0}^{2 \pi} \mathrm{d} \theta_{k} \prod_{\alpha=1}^{b N} \int_{0}^{2 \pi} \mathrm{d} \theta_{\alpha} \times \\
& \times \prod_{1 \leqslant j<k \leqslant a N}\left|\mathrm{e}^{i \theta_{j}}-\mathrm{e}^{i \theta_{k}}\right|^{2} \prod_{1 \leqslant \alpha<\beta \leqslant b N}\left|\mathrm{e}^{i \theta_{\alpha}}-\mathrm{e}^{i \theta_{\beta}}\right|^{4} \prod_{j=1}^{a N} \prod_{\alpha=1}^{b N}\left|\mathrm{e}^{i \theta_{j}}-\mathrm{e}^{i \theta_{\alpha}}\right|^{2} .
\end{aligned}
$$

For evaluating $Z$, we express its integrand as a phase factor

$$
(-1)^{(a+b) N} \prod_{k=1}^{a N} \mathrm{e}^{-i b N \theta_{k}} \prod_{\alpha=1}^{b N} \mathrm{e}^{-i[(a+2 b) N-2] \theta_{\alpha}}
$$


multiplied by

and

$$
A_{1}=\prod_{1 \leqslant j<k \leqslant a N}\left(\mathrm{e}^{-i \theta_{k}}-\mathrm{e}^{-i \theta_{j}}\right)
$$

$$
A_{2}=\prod_{1 \leqslant j<k \leqslant a N}\left(\mathrm{e}^{i \theta_{k}}-\mathrm{e}^{i \theta_{j}}\right) \prod_{j=1}^{a N} \prod_{\alpha=1}^{b N}\left(\mathrm{e}^{i \theta_{j}}-\mathrm{e}^{i \theta_{\alpha}}\right)^{2} \prod_{1 \leqslant \alpha<\beta \leqslant b N}\left(\mathrm{e}^{i \theta_{\beta}}-\mathrm{e}^{i \theta_{\alpha}}\right)^{4} .
$$

$A_{1}$ is a Vandermonde determinant, which has the expansion

$$
A_{1}=\sum_{Q=1}^{(a N) !} \varepsilon(Q) \prod_{j=1}^{a N} \mathrm{e}^{-i \theta_{j}[Q(j)-1]},
$$

where $Q$ is a permutation of the indices which transforms $j$ into $Q(j)$ and the signature of which is $\varepsilon(Q)$. The product $A_{2}$ occurs in another solvable model [8]; it can be expressed as a confluent alternant determinant, with the expansion

$$
\begin{aligned}
A_{2}=\sum_{X} \varepsilon(P) \prod_{l=1}^{a N} & \mathrm{e}^{i \theta_{l}[P(l)-1]} \times \\
& \times \prod_{\alpha=1}^{b N}[P(a N+2 \alpha)-P(a N+2 \alpha-1)] \mathrm{e}^{i \theta_{\alpha}[P(a N+2 \alpha)+P(a N+2 \alpha-1)-3]},
\end{aligned}
$$

where $X$ denotes the set of all permutations $P$ of $\{1,2, \ldots,(a+2 b) N\}$ such that $P(2 l)>$ $P(2 l-1)$ for each $l=1,2, \ldots, N(b+a / 2)$ (we thus require $a N$ to be even). Hence

$$
\begin{aligned}
Z=R^{-(a+2 b) N}(-1)^{(a+b) N} & \sum_{X} \varepsilon(P) \sum_{Q=1}^{(a N) !} \varepsilon(Q) \prod_{l=1}^{a N} u_{P(l), Q(l)} \times \\
& \times \prod_{l=1}^{b N}[P(a N+2 l)-P(a N+2 l-1)] v_{P(a N+2 l-1), P(a N+2 l)},
\end{aligned}
$$

where

and

$$
u_{P(l), Q(l)}=\frac{1}{2 \pi} \int_{0}^{2 \pi} \mathrm{d} \theta \mathrm{e}^{i \theta[P(l)-Q(l)-b N]}
$$

$$
v_{P(a N+2 l-1), P(a N+2 l)}=\frac{1}{2 \pi} \int_{0}^{2 \pi} \mathrm{d} \theta \mathrm{e}^{i \theta[P(a N+2 l)+P(a N+2 l-1)-1-(a+2 b) N]} .
$$

$u$ and $v$ are equal to 1 when the coefficient of $\theta$ in the exponential vanishes, and otherwise equal to 0 . Since $Q(l) \in\{1,2, \ldots, a N\}$, we require

$$
P(l) \in\{b N+1, b N+2, \ldots,(a+b) N\}, \quad l=1,2, \ldots, a N
$$

for non-zero contributions to the partition function.

Further, the only permutations satisfying $P(a N+2 l)+P(a N+2 l-1)=(a+2 b) N$ +1 and $P(a N+2 l)>P(a N+2 l-1), l=1,2, \ldots, b N$ are

$$
P(a N+2 l-1)=R(l), \quad P(a N+2 l)=(a+2 b) N+1-R(l),
$$

where $R(l)$ is a permutation on $\{1,2, \ldots, b N\}$, and each such permutation has the same parity. Hence

$$
Z=R^{-(a+2 b) N} \sum_{Q=1}^{(a N) !(b N) !} \sum_{R=1}^{b N} \prod_{l=1}^{b N}[(a+2 b) N+1-2 R(l)]
$$


But in (4.11) all permutations $Q$ and $R$ give the same contribution, so we can choose $R(l)=l$ and thus obtain

$$
Z=R^{-(a+2 b) N} \frac{(2 b N+a N) !(a N / 2) !(b N) !}{2^{b N}(b N+a N / 2) !}
$$

In the thermodynamic limit, $N, R \rightarrow \infty$, for fixed values of the densities $\rho_{\mathrm{R}}=a N / 2 \pi R$ and $\rho_{\mathrm{G}}=b N / 2 \pi R$, using Stirling's formula, one obtains an excess free energy density $f$ such that

$$
\begin{aligned}
\beta f=-\lim \frac{\ln Z}{2 \pi R}=\rho_{\mathrm{R}}+2 \rho_{\mathrm{G}} & +\frac{1}{2} \rho_{\mathrm{R}} \ln \frac{\left(\rho_{\mathrm{R}}+\rho_{\mathrm{G}}\right)^{2}}{\rho_{\mathrm{R}}\left(\rho_{\mathrm{R}}+2 \rho_{\mathrm{G}}\right)}+ \\
& +\rho_{\mathrm{G}} \ln \frac{\left(\rho_{\mathrm{R}}+\rho_{\mathrm{G}}\right)^{2}}{\rho_{\mathrm{G}}\left(\rho_{\mathrm{R}}+2 \rho_{\mathrm{G}}\right)}-\left(\rho_{\mathrm{R}}+2 \rho_{\mathrm{G}}\right) \ln \left[2 \pi\left(\rho_{\mathrm{R}}+\rho_{\mathrm{G}}\right)\right] .
\end{aligned}
$$

Thus, the thermodynamic limit does exist. If one introduces a length scale $L$ for the potentials, defining them with a $\ln (r / L)$ instead of $\ln r$, the logarithm in the last term of $(4.13)$ becomes $\ln \left[2 \pi\left(\rho_{R}+\rho_{G}\right) L\right]$, which is the logarithm of a dimensionless quantity, as it should.

4.2 Correlation FUnCtions. - The two-body distribution functions can be evaluated by the same method as the partition function, except that the book-keeping of the permutations which give non-zero contributions is more complicated. One finds double sums of non-zero contributions, which, in the thermodynamic limit, become double integral representations for the distribution functions. The corresponding correlation functions $h_{\mathrm{AB}}(r)$, where $r$ is the distance between the two particles, are

$$
\begin{aligned}
& h_{\mathrm{RR}}(r)=-\frac{\sin ^{2}\left(\pi \rho_{\mathrm{R}} r\right)}{\left(\pi \rho_{\mathrm{R}} r\right)^{2}}-\frac{b}{a} \int_{0}^{1} \mathrm{~d} t \int_{0}^{1} \mathrm{~d} s \frac{1-2 s}{2 b t / a+1}\left(\mathrm{e}^{2 \pi i \rho_{\mathrm{R}} r(s-1-b t / a)}-\mathrm{e}^{2 \pi i \rho_{\mathrm{R}} r(s+b t / a)}\right) \text {, } \\
& h_{\mathrm{GG}}(r)=\int_{0}^{1} \mathrm{~d} t \int_{0}^{1} \mathrm{~d} s\left\{-\frac{(t+s+a / b)^{2}}{(2 t+a / b)(2 s+a / b)} \mathrm{e}^{2 \pi i \rho_{\mathrm{G} r}(t-s)}+\right. \\
& \left.+\frac{(t-s)^{2}}{(2 t+a / b)(2 s+a / b)} \cos \left[2 \pi \rho_{\mathrm{G}} r(t+s+a / b)\right]\right\} \\
& h_{\mathrm{RG}}(r)=-2 \int_{0}^{1} \mathrm{~d} t \int_{0}^{1} \mathrm{~d} s \frac{t+a s / b}{2 t+a / b} \cos \left[2 \pi \rho_{\mathrm{R}} r(b t / a-s+1)\right] .
\end{aligned}
$$

Obviously, these expressions could be rewritten in terms of simple integrals only.

One can readily check that the screening rules (2.9) are satisfied. The asymptotic behaviours, as $r \rightarrow \infty$, are

$$
\begin{aligned}
& h_{\mathrm{RR}}(r) \sim-\frac{1}{\left(\pi \rho_{\mathrm{R}} r\right)^{2}}+\frac{\cos \left(2 \pi \rho_{\mathrm{G}} r\right)+\cos \left[2 \pi\left(\rho_{\mathrm{R}}+\rho_{\mathrm{G}}\right) r\right]}{2\left(\pi \rho_{\mathrm{R}} r\right)^{2}\left(1+\frac{2 b}{a}\right)}+\mathcal{O}\left(\frac{1}{r^{3}}\right) \\
& h_{\mathrm{GG}}(r) \sim-\frac{1}{2\left(\pi \rho_{\mathrm{G}} r\right)^{2}}+\frac{\left(2+\frac{a}{b}+\frac{b}{a}\right) \cos \left(2 \pi \rho_{\mathrm{G}} r\right)+\frac{b}{a} \cos \left[2 \pi\left(\rho_{\mathrm{R}}+\rho_{\mathrm{G}}\right) r\right]}{2\left(\pi \rho_{\mathrm{G}} r\right)^{2}\left(2+\frac{a}{b}\right)}+\mathcal{O}\left(\frac{1}{r^{3}}\right)
\end{aligned}
$$




$$
h_{\mathrm{RG}}(r) \sim \frac{1}{2 \rho_{\mathrm{R}} \rho_{\mathrm{G}}(\pi r)^{2}}+\frac{\cos \left[2 \pi\left(\rho_{\mathrm{R}}+\rho_{\mathrm{G}}\right) r\right]-\left(1+\frac{a}{b}\right) \cos \left(2 \pi \rho_{\mathrm{G}} r\right)}{2 \rho_{\mathrm{R}} \rho_{\mathrm{G}}(\pi r)^{2}\left(2+\frac{a}{b}\right)}+\mathcal{O}\left(\frac{1}{r^{3}}\right) .
$$

The non oscillating terms are indeed in agreement with (3.3). In addition, there are oscillating terms of an interesting form. They decay (algebraically) at large distances (and thus there is no long-range order), but at finite distances they exhibit two (in general incommensurable) periods : $\left(\rho_{\mathrm{R}}+\rho_{\mathrm{G}}\right)^{-1}$ and $\rho_{\mathrm{G}}^{-1}$. Thus, like in other logarithmic one-dimensional models $[9,10]$, there is a local tendency to crystalline ordering, but here there is a competition between a period determined by the total density and a period determined by the partial density of the more strongly coupled greek particles. The period $\rho_{\mathbf{R}}^{-1}$ also appears, in higher order terms (at the order $1 / r^{4}$ ).

\section{Summary and conclusion.}

The pair correlation functions obtained from the two-dimensional trial wave function (1.1) must obey the sum rules (2.9) and (2.10). The one-dimensional analogs of these rules can be checked on a solvable one-dimensional model, which we believe to be also intrinsically of some interest.

\section{Acknowledgments.}

One of us (B. J.) is indebted to D. Levesque for having suggested to use the asymptotic behaviour of the direct correlation functions, and to E. Brézin and C. Itzykson for having brought Girvin's preprint to our attention.

\section{References}

[1] Tsui, D. C., Stormer, H. L. and Gossard, A. C., Phys. Rev. Lett. 48 (1982) 1559.

[2] Stormer, H. L., Chang, A., Tsui, D. C., Huang, J. C. M., Gossard, A. C. and Wiegmann, W., Phys. Rev. Lett. 50 (1983) 1953.

[3] Laughlin, R. B., Phys. Rev. Lett. 50 (1983) 1395.

[4] Halperin, B. I., Helv. Phys. Acta 56 (1983) 75.

[5] GiRvin, S. M., National Bureau of Standards preprint.

[6] Stillinger, F. H. and Lovett, R., J. Chem. Phys. 49 (1968) 1991.

[7] See e.g. Baus, M. and Hansen, J. P., Phys. Rep. 59 (1980) 1.

[8] Forrester, P. J., J. Austr. Math. Soc. B, to be published.

[9] Mehta, M. L., Random Matrices (Academic, New York) 1967.

[10] Forrester, P. J., J. Stat. Phys., to be published. 